HD-THEP-98-48

\title{
SUSY Variants of the Electroweak Phase Transition
}

\author{
Stephan J. Huber \\ Michael G. Schmidt \\ Institut für Theoretische Physik \\ Universität Heidelberg \\ Philosophenweg 16, D-69120 Heidelberg, FRG
}

\begin{abstract}
The MSSM with a light right-handed stop and supersymmetric models with a singlet whose vev is comparable to that of the $S U(2)_{W}$ Higgs allow for a strongly first-order electroweak phase transition even for a mass of the lightest Higgs around $100 \mathrm{GeV}$. After a short review of the standard model situation we discuss these supersymmetric models. We also compare perturbative calculations based on the dimensionally reduced 3-dimensional action with lattice results and present an analytic procedure based on an analogue of the stochastic vacuum model of QCD to estimate the nonperturbative contributions.
\end{abstract}

*Based on a talk presented by M. G. Schmidt at the First European Meeting "From the Planck scale to the electroweak scale" (Kasimierz, Poland, May 1998) 


\section{Introduction}

The unification of fundamental interactions at high energies is usually discussed in the framework of the renormalization group based on perturbation theory for temperature $T=0$ quantum field theory. However, according to our present understanding, high energies much above the reach of today's accelerators were realized in the hot early universe: during its expansion it cooled down and - like in material physics (liquid-vapor, alloys, superconductors...) underwent phase transitions. The latter depend crucially on the particle content and the interactions of the underlying theory and on the time scale of the expanding universe. Thus the study of possible relics of such phase transitions might reveal interesting news about the basic theory. In general, these are genuinely nonperturbative phenomena. Therefore one needs methods to treat them appropriately.

The electroweak standard model (SM) is so successful because it allows for very accurate perturbative calculations of high energy processes due to the small weak coupling $g_{W}$ in the Higgs ground state and because these agree beautifully with experiments. Still it is not considered as a fundamental theory above the $1 \mathrm{TeV}$ scale because it is not stable against impact from GUT-scale physics. Thus any hint towards a modification of the electroweak standard model is highly welcome to theorists.

We will first review shortly the well understood phase transition (PT) in the SM in chapter 2. It has all the necessary ingredients postulated by Sakharov to possibly produce [1, 2] the baryon asymmetry of the universe $\left(\left(n_{B}-n_{\bar{B}}\right) / n_{\gamma} \sim 10^{-10}\right)$, and this is the main promise of a standard model PT. However, it turned out to be not fulfilled. Thus in the discussion of baryogenesis one might have to return to the old (or new) out-of-equilibrium decay scenarios [3] of very heavy particles contained in GU models. However, grand unification should be dealt with in close relation to inflation: inflation is the well-known solution to some problems in standard cosmology and also provides a very successful explanation of primordial density fluctuations in the early universe. Since all particle densities are diluted exponentially during the inflationary period, the GUT particles supposed to create some baryon asymmetry have to be produced in the pre-reheating period after inflation or in a separate GUT phase transition after inflation. The latter is very problematic because the reheating temperature after inflation is favourably not in the range of GUT energies, in particular in SUSY models. Furthermore, the GU theory has to be $B-L$ violating, otherwise $\mathrm{B}$ and $\mathrm{L}$ are washed out by the $B-L$ conserving (hot) sphaleron transitions during the thermodynamic equilibrium period before the electroweak PT (fig. 1).

Figure 1:

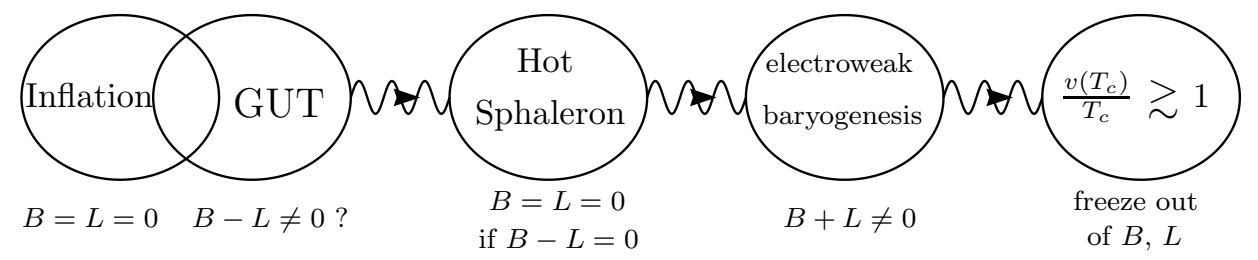


Chapter 3 contains some remarks about a (semi)analytic treatment of nonperturbative effects in a first-order PT based on recent work of the authors. The most important point is an instability of the gauge field $F^{2}=0$ vacuum of the hot electroweak theory for small Higgs vevs. This leads to nonperturbative modifications of the electroweak potential. In chapter 4 and 5 we discuss variants of the SM: in chapter 4 the minimal supersymmetric standard model (MSSM) with a "light" $s p_{R}$ superpartner of the right-handed top. This will allow a strongly first-order phase transition even at Higgs masses as large as $100 \mathrm{GeV}$. In chapter 5 we consider a nonminimal (next to minimal?) supersymmetric model (NMSSM) with a singlet superfield $S$ which also obtains a vev $\langle S\rangle$ of the order of the Higgs vev. Different from the standard situation this model leads to a first-order phase transition already at the tree level. Again Higgs masses of $100 \mathrm{GeV}$ are compatible with a strong PT in a broad range of parameters.

\section{Electroweak Phase Transition in the Standard Model}

The order and the strength of the electroweak PT can be discussed with the (thermal equilibrium) effective potential $V_{\text {eff }}\left(\varphi^{2}, T\right)$ (free energy) depending on the Higgs background field $\varphi\left(\langle\phi\rangle=\left(\begin{array}{l}\varphi \\ 0\end{array}\right) / \sqrt{2}\right)$ and the temperature. A simple 1-gauge field loop calculation in thermal field theory results in a positive (Debye) mass contribution to the Higgs mass proportional to $T$. This reduces the Higgs instability and predicts a phase transition at high temperatures [4]. The Matsubara zero modes (without time dependence) in the same 1-loop calculation produce a term $\sim-T\left(\varphi^{2}\right)^{3 / 2}$ in the potential which leads to two degenerate minima at some critical temperature $T_{c}$ labelled by "symmetric" $(\varphi=0)$ and "Higgs" $\left(\varphi_{\text {Min }} \neq 0\right)$. This would naively imply a first-order PT [5]. However, a concise analysis of the effective potential reveals that this is a very preliminary result because one has to treat the infrared (IR) behavior for $\varphi \rightarrow 0$ properly. In high-temperature gauge theory the coupling $g_{W}^{2} T$ has a dimension, and the dimensionless ratio $g_{W}^{2} / m_{I R}$ is not small if the scale $m_{I R} \sim \varphi$ becomes small. Furthermore it turns out that 2-loop contributions are quantitatively very important. A clean way [7, 8] to deal with such a situation is a stepwise procedure ("dimensional reduction"): Integrate out

1) $n \neq 0$ Matsubara modes with $p_{0_{n}}=2 \pi n T$ (including all fermions with $n=$ $1 / 2,3 / 2, \ldots)$.

2) $n=0$ modes of longitudinal gauge fields $A_{0}$ which have obtained a Debye mass $m_{D} \sim g_{W} T$ in the first step.

Here "integrate out" is understood in the sense of a matching procedure, matching a set of static 4-dimensional amplitudes containing the above modes in the loops to a 3-dimensional truncated Lagrangian [9] for the Higgs and transversal gauge zero modes:

$$
L_{e f f}^{3-d i m}=\frac{1}{4}\left(F_{i k}^{a}\right)^{2}+\left(D_{i} \phi_{3}\right)^{+}\left(D_{i} \phi_{3}\right)+m_{3}^{2}(T) \phi_{3}^{+} \phi_{3}+\lambda_{3}(T)\left(\phi_{3}^{+} \phi_{3}\right)^{2} .
$$




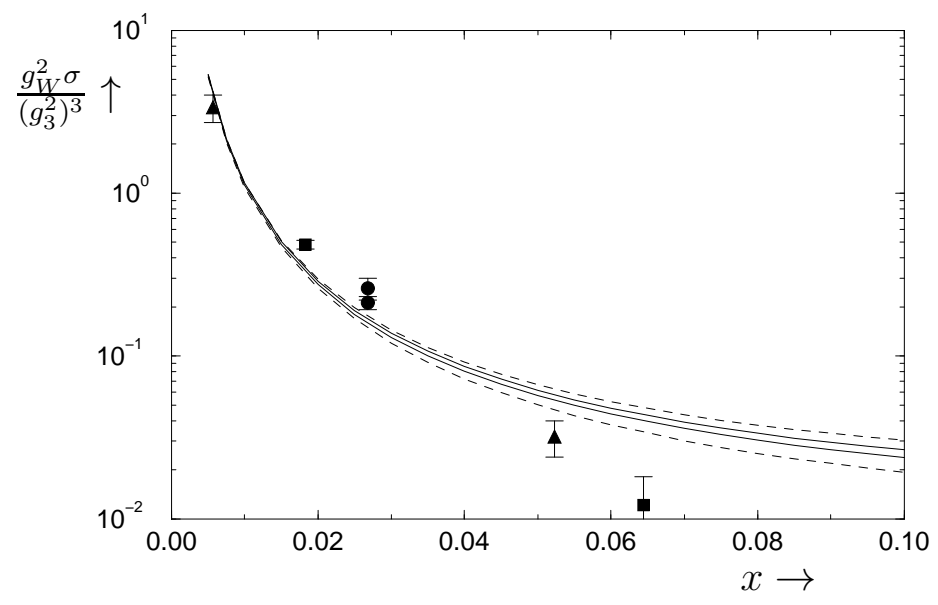

Figure 2: (from ref. [15]). The perturbatively calculated interface tension $\sigma$ (including $Z$-factor effect and gauge variations) vs. $x$ compared to lattice data from ref. [10] (squares), ref. [16] (triangles) and ref. [17] (circles).

$L_{\text {eff }}^{3-\text { dim }}$ contains a 3 -dimensional gauge coupling $g_{3}^{2}=g_{W}^{2} T(1+\ldots)$, a $T$-dependent Higgs mass $m_{3}^{2}(T)$ and a coupling $\lambda_{3}(T)=\lambda_{T}^{4-d i m} T$ between 3-dimensional Higgs fields (canonical dimension 1/2). Neglection of higher terms in $(2.1)\left(\right.$ e.g. $\left.\sim\left(\phi_{3}^{+} \phi_{3}\right)^{3}\right)$ introduces a (few percent) error of $\mathcal{O}\left(g_{W}^{3}\right)$ for $\varphi_{3}<2 \pi \sqrt{T}$. It is important to note that steps 1 and 2 above can be performed in (two-loop) perturbation theory, whereas the zero-mode Lagrangian (2.1) contains all the IR problems for $\varphi \rightarrow 0$. As it stands $L_{\text {eff }}^{3-d i m}$ contains a potential which still naively describes a secondorder PT at $T_{c}$ with $m_{3}^{2}\left(T_{c}\right)=0$. However, it is just the tree Lagrangian of the 3 -dimensional theory. Calculating naively again the 1-loop action in this theory one reproduces the $-T\left(g_{W}^{2} \varphi^{2}\right)^{3 / 2}$ term mentioned above (now in the form $-\left(g_{3}^{2} \varphi_{3}^{2}\right)^{3 / 2}$ ) leading to a first-order PT. However, we expect important nonperturbative IR effects if the perturbative 3-dimensional potential competes with a nonperturbative part at $\varphi_{3}=0$ which is of order $\left(g_{3}^{2}\right)^{3}$ for dimensional reasons. Rescaling by powers of $g_{3}^{2}-$ the unique scale in the problem - we have the dimensionless couplings

$$
y=\frac{m_{3}^{2}(T)}{\left(g_{3}^{2}\right)^{2}} \quad, \quad x=\frac{\lambda_{3}(T)}{g_{3}^{2}}\left(\sim \frac{\lambda_{T}^{4-d i m}}{g_{W}^{2}}\right)
$$

where $y$ is related to $\left(T-T_{c}\right)$ and $x$ is the parameter which determines the critical behaviour of (2.1).

The most secure way to deal with (2.1) is to use it in lattice calculations 10, 11] to determine the critical temperature $T_{c}$ and the interface tension and latent heat if we have a first-order PT. An alternative treatment would be in the framework of Wilsonian renormalization [12]. $L_{e f f}^{3-d i m}$ of eq. (2.1) is an $x, y$-dependent superrenormalizable 3-dimensional Lagrangian with just one scale $g_{3}^{2}$ and without fermions and thus can be handled very safely in the lattice approach. The results of such lattice calculations [10, 11] are:

(i) there is a first-order PT for $x \lesssim 0.11$; there is a second-order PT at the endpoint [14] and above $x=0.11$ one has a crossover - no PT anymore! 


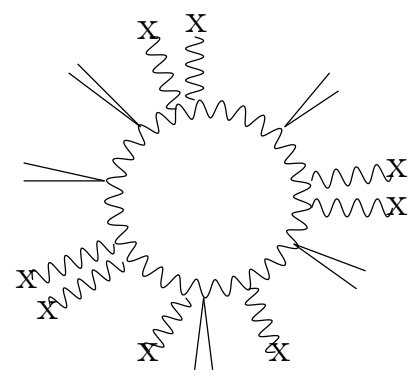

Figure 3: 1-loop graph contributing to the potential $V\left(\varphi^{2},<g_{3}^{2} F^{2}>\right)$.

(ii) $v\left(T_{c}\right) / T_{c}=\varphi_{\min }\left(T_{c}\right) / T_{c} \gtrsim 1$ for $x \lesssim 0.04$

(iii) Comparing the 2-loop perturbative expressions obtained from (2.1) with lattice results, there are deviations for $x \gtrsim 0.05$ in particular for the interface tension (fig. 2).

To protect a previously generated baryon asymmetry in a universe with $B-L=0$ from erasure by sphaleron transitions $\sim \exp (-A v(T) / T)$ in a thermodynamic equilibrium period inside the Higgs phase one needs $v\left(T_{c}\right) / T_{c} \gtrsim 1$. With $\lambda=(1 / 8) m_{H}^{2} / m_{W}^{2}+c_{P o s} m_{t}^{4} / m_{W}^{4}$ where the second term alone is $>0.04$ for the observed top mass $m_{t}$, this can never be achieved in the SM, independent of the Higgs mass. Together with its CP-violating effects being smaller than needed for an asymmetry production, this prevents the SM to explain the baryon asymmetry of the universe.

\section{Nonperturbative effects in the three-dimensional electroweak potential}

Lattice results give a clear picture for the phase diagram in the case of Lagrangian (2.1). However, for some questions - e.g. sphaleron action, shape and action of the critical bubble - an explicit effective (coarse-grained) action still would be useful. It is also very important to have some (semi)analytic picture which tells us where one can trust perturbation theory and where not. This will be particularly true in the case of more complicated effective actions where lattice results may not be available. Thus we shortly discuss such a model [13].

In the hot symmetric phase with background $\varphi=0$ the Lagrangian (2.1) describes a 3-dimensional QCD-type theory with scalar Higgs "quarks". Lattice calculations 11] show that indeed in this phase static "quarks" experience a constant string tension which furthermore is approximately equal to that of pure $\mathrm{SU}(2)$-YangMills theory. This hints to a nonperturbative dynamics dominated by "W-gluons". Also a spectrum of correlation masses of gauge-invariant $H \bar{H}$ bound states and of $W$-glueballs has been calculated on the lattice [18]. The former is compatible with a linearly rising potential in a relativistic bound state model [19] (like that of Simonov 


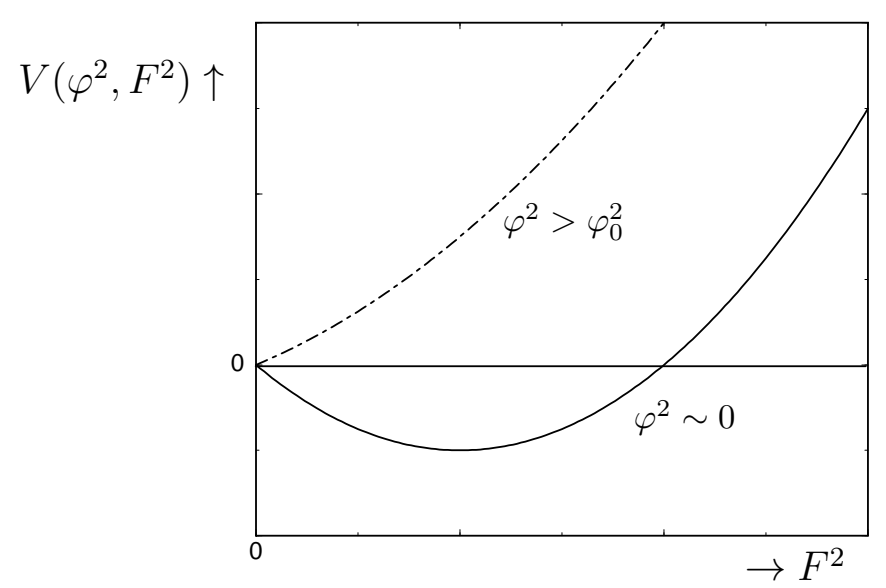

Figure 4: Sketch of the potential $V\left(\varphi^{2},<g_{3}^{2} F^{2}>\right)$ in $F^{2}$-direction for two different values of $\varphi^{2}$.

in 4-dimensional QCD [20]). There is only a small mixing with the W-glueballs [18] in agreement with the suggestion above that we have pure "W-gluon" dynamics.

An interesting phenomenological description of the QCD vacuum is the "stochastic vacuum model" of Dosch and Simonov [21, 22]. Its main virtue is that it leads very naturally to the area law of confinement. We have applied it to the 3-dimensional theory (2.1) with an $S U(2)_{W}$ gauge group. Its main ingredient is a correlated gauge field background with a purely Gaussian correlation

$$
\ll g_{3}^{2} F_{i \kappa}^{a}\left(x^{\prime}\right) F_{i \kappa}^{a}(x) \gg=<g_{3}^{2} F^{2}>D\left(\frac{\left(x-x^{\prime}\right)^{2}}{a^{2}}\right) .
$$

This correlator is already simplified by choice of a coordinate gauge and by averaging over the tensor structure. $\left\langle g_{3}^{2} F^{2}\right\rangle$ is the normalization by the usual local gauge field condensate and $D(D(0)=1)$ is a form factor containing the correlation length $a$. The correlator has been tested in 3-dimensional lattice calculations [23] and the correlation length was obtained as $a \sim 1 / 0.73 g_{3}^{2} \sim 2 / m_{\text {glueball }}$. In ref. 13 we presented strong indications that the $\left\langle g_{3}^{2} F^{2}>\right.$ ground state is unstable (similar to the Savvdidy instability of QCD) for small Higgs vevs. Thus one obtains nonperturbative effects by a fluctuating gauge field background of type (3.1).

One can estimate the effect of such a background on the W-boson (and ghost) loop leading to the 1-loop effective potential $V\left(\varphi^{2},<g_{3}^{2} F^{2}>\right.$ ) (fig. 3). We found [13] two contributions to a momentum-dependent effective ("magnetic") mass:

(i) an IR regulator mass $m_{\text {conf }}^{2}\left(p^{2}, \varphi^{2},<g_{3}^{2} F^{2}>\right.$ ) of gauge bosons and ghosts due to the string tension (area law) which cures the IR problems of perturbation theory.

(ii) a negative effective (mass) ${ }^{2}$ for the W-bosons $-\tilde{S}_{F}\left(p^{2}, \varphi^{2},<g_{3}^{2} F^{2}>\right)$ due to spin-spin forces which becomes important for larger $p^{2}$ ("paramagnetism") and does not spoil the nice IR properties of $m_{\text {conf }}^{2}$. If we introduce these masses in the 1-loop action (gauge boson loop) it has roughly the form 

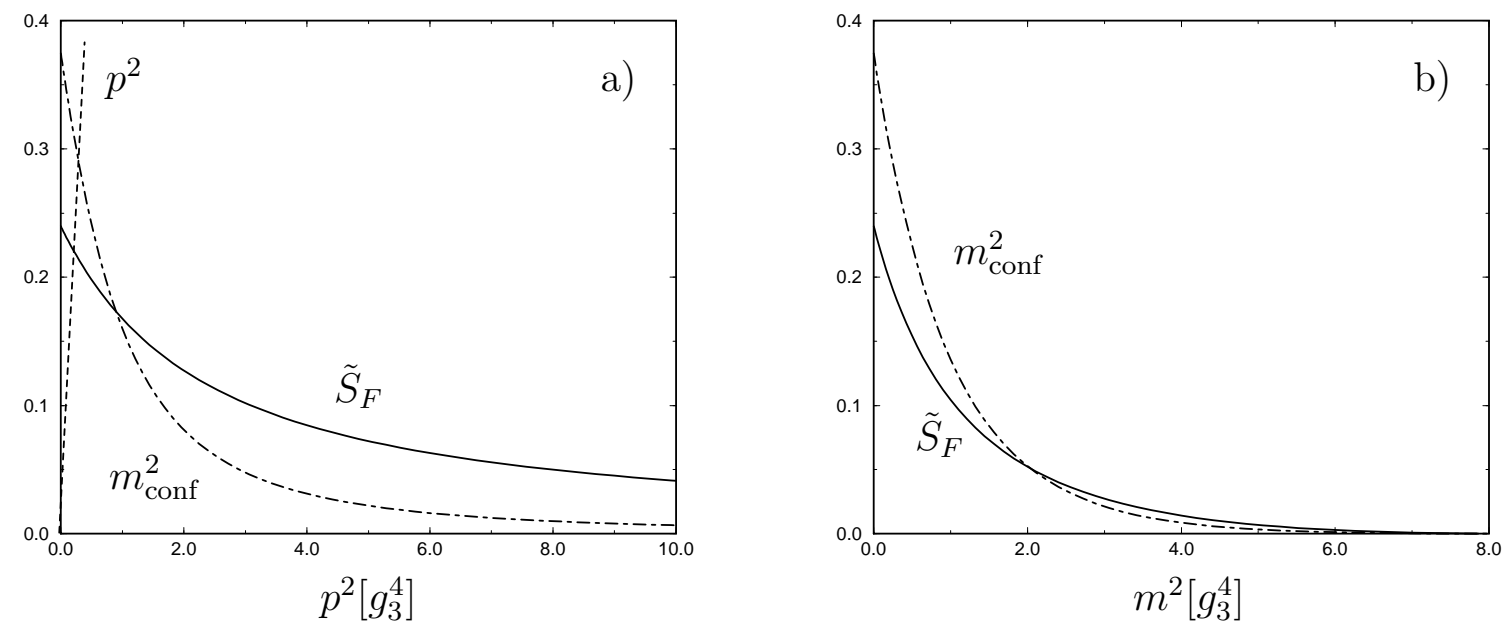

Figure 5: (from ref. [13]). $m_{\text {conf }}^{2}\left(p^{2}, m^{2}\right)$ and $\tilde{S}_{F}\left(p^{2}, m^{2}\right)$ in units of $\left(g_{3}^{2}\right)^{2}$ plotted a) for $m^{2}=0$ and b) $p^{2}=0$.

a)

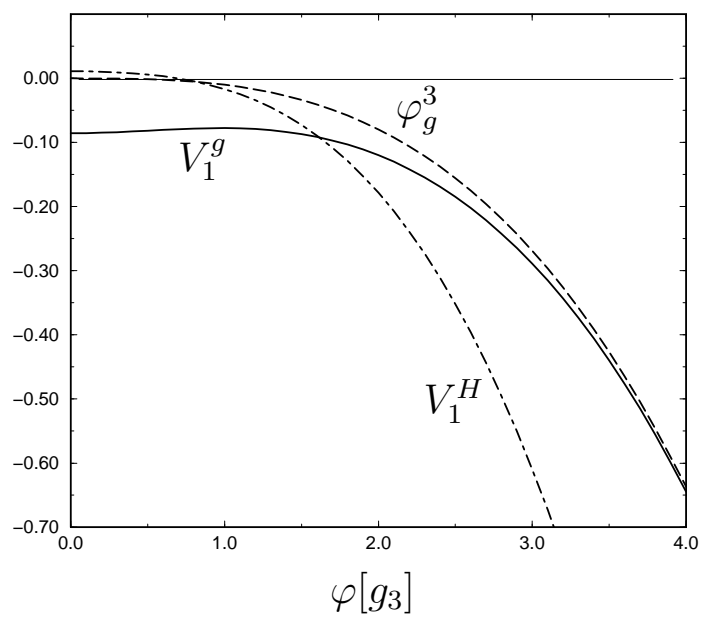

b)

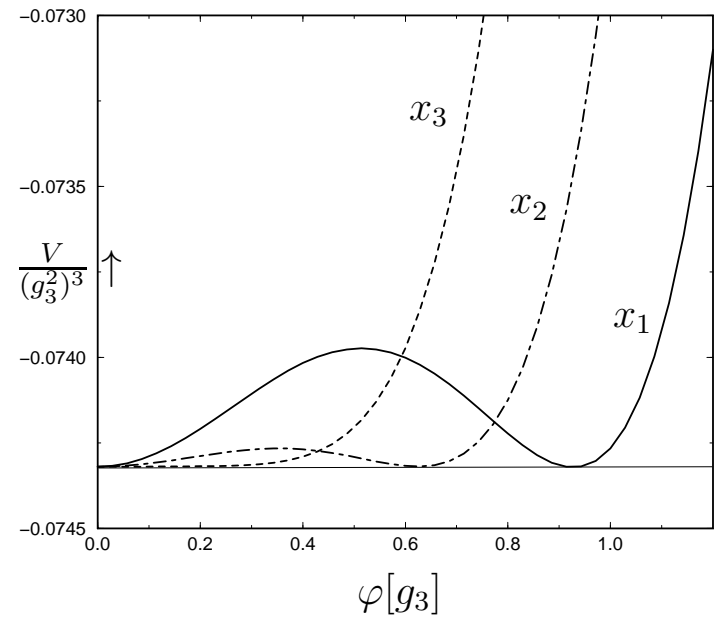

Figure 6: (from ref. [13]). a) $V_{1}^{g}(\varphi), V_{1}^{H}(\varphi)$ compared to the perturbative $\varphi_{g}^{3}$ term (in units of $\left.\left(g_{3}^{2}\right)^{3}\right)$. b) Fading away of the first-order phase transition with increasing $x=\frac{\lambda}{g_{3}^{2}}$, where $x_{1}=0.06, x_{2}=0.08$ and $x_{3}=0.11$. 


$$
\begin{aligned}
V\left(\varphi^{2},<g_{3}^{2} F^{2}>\right) \sim & \ldots \int \frac{d^{3} p}{(2 \pi)^{3}} \log \left[p^{2}+\frac{1}{4} g_{3}^{2} \varphi^{2}+m_{\text {conf }}^{2}\left(p^{2}, \varphi^{2},<g_{3}^{2} F^{2}>\right)\right. \\
& \left.-\tilde{S}_{F}\left(p^{2}, \varphi^{2},<g_{3}^{2} F^{2}>\right)\right] .
\end{aligned}
$$

(This has to be corrected 13 for combinatorics and also has to be renormalized). Both masses depend on $\left\langle g_{3}^{2} F^{2}\right\rangle$. Expanding (3.2) in first-order in $\left\langle g_{3}^{2} F^{2}\right\rangle$ the spin-spin force in $-\tilde{S}_{F}$ produces the well-known negative $F^{2}$-term destabilizing the $F^{2}=0$ vacuum. Adding the tree $\frac{1}{4} F^{2}$ we can obtain an effective potential sketched in fig. Tt stabilized at some value $F^{2} \neq 0$ by confinement forces. This is a 1-loop calculation and the masses $m_{\text {conf }}^{2}$ and $-\tilde{S}_{F}$ are determined only roughly (in lack of lattice data support). Thus we have only a qualitative picture. To proceed, we fixed $<g_{3}^{2} F^{2}>$ at the minimum by a relation to the lattice string tension.

Fig. 5 shows the qualitative form of $m_{\text {conf }}^{2}\left(p^{2}, \varphi^{2}\right)$ and of $\tilde{S}_{F}\left(p^{2}, \varphi^{2}\right)$ and fig. 6 a the modified " $\varphi$ " -term corresponding to (3.2). Fig. 6b presents the new 1loop potential at the critical temperature at various $x$-values, and one can see the first-order PT fading away. One can also evaluate the interface tension (table) and determine roughly the crossover point by postulating that the effective $\varphi^{2}$ and $\varphi^{4}$ vanish at this (conformal) point with a second-order PT.

\begin{tabular}{|c||c|c|}
\hline$x$ & $\sigma$ & $\sigma_{\text {perturbative }}$ \\
\hline \hline 0.06 & 0.016 & 0.013 \\
\hline 0.08 & 0.004 & 0.007 \\
\hline 0.11 & 0 & 0.004 \\
\hline
\end{tabular}

We should stress again that this picture of nonperturbative effects is not really quantitative, in particular because 2-loop calculations in a correlated gauge-field background are (too) difficult. Still we might get an indication in which direction nonperturbative contributions go.

\section{The MSSM with a "light" stop}

Searching for modifications of the electroweak theory in order to obtain a strongly first-order PT, one faces the by now sufficiently known situation that the success of the standard model is both blessing and burden. We do not have experimental hints which way to go. Supersymmetric theories have the well-known theoretical advantages. From a practical point of view all one needs for a strongly first-order PT is the strengthening of the " $\varphi$ " -term in the effective potential due to bosonic exchange in the loop. Thus one needs further bosons with a strong coupling to the Higgs. SUSY models have a host of new bosons in the superpartner sector. In particular the $s$-top particles have a particularly strong Yukawa coupling $h_{t}$ if the Higgs vev $<v_{2}>$ of the Higgs coupling to the top $\left(m_{t o p}=h_{t}<v_{2}>\right)$ is not very large, i.e. if $\tan \beta=v_{2} / v_{1}$ is not large. The superpartner of the right-handed top, the stop $_{R}$, does not have $S U(2)_{W}$ interactions, and thus is particularly flexible in its allowed mass (no $\rho$-parameter problem). As proposed in ref. [24, 25], its exchange 


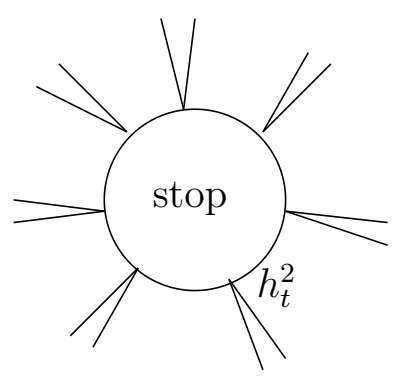

Figure 7: 1-loop stop contribution to the effective potential.

(fig. 7) can enhance the PT significantly if its mass $m_{3}^{2}$ in the symmetric phase (including $T^{2}$-plasma mass) is small:

$$
m_{3}^{2}=m_{0}^{2}+c T^{2}
$$

where $m_{0}^{2}$ is the SUSY-breaking scalar mass of the $s_{0} p_{R}$. The $T=0$ mass of the stop $_{R}$ is

$$
m_{\tilde{t}}^{2}=m_{0}^{2}+m_{t}^{2}
$$

and is not much larger than the top mass for small positive $m_{0}^{2}$. There might be a nonuniversal SUSY mass breaking at the GUT scale necessary for very small $m_{0}^{2}$ though the stop mass ${ }^{2}$ is naturally lowered by renormalization flow.

If the $s t_{R}$ and one heavy combination of Higgses is integrated out, one is led again to a Lagrangian of the form (2.1), but now with an $x=\lambda_{3} / g_{3}^{2}$ value much smaller than in the SM (being bosonic the stop contributes opposite to the top!) allowing for $m_{H} \lesssim 75 \mathrm{GeV}$ for a strongly first-order PT with $v\left(T_{c}\right) / T_{c} \geq 1$ [27]-[30].

One can also ask [24] for $s t o p_{R}$ masses smaller than the top mass taking $m_{0}^{2}=$ $-\tilde{m}_{0}^{2}$ negative in (4.1), (4.2). The $\operatorname{stop}_{R}$ than should not be fully (also zero modes) integrated out, but kept in the effective 3-dimensional zero mode action together with the light Higgs fields. If one assumes that the CP-odd Higgs $A_{0}$ meson surviving spontaneous breaking is rather heavy $(\gtrsim 300 \mathrm{GeV})$, there is a heavy Higgs sector to be integrated out, and just as above one Higgs field remains. We thus have to consider a Lagrangian 28]

$$
\begin{aligned}
L_{\text {eff }}^{3-d i m}= & L_{\text {eff }}^{3-\text { dim }}(\text { Higgs }) \\
& +\frac{1}{4} G_{i j}^{A} G_{i j}^{A}+\left(D_{i}^{s} U\right)^{+}\left(D_{i}^{s} U\right)+m_{U_{3}}^{2} U^{+} U \\
& +\lambda_{U_{3}}\left(U^{+} U\right)^{2}+\gamma_{3}\left(\phi_{3}^{+} \phi_{3}\right)\left(U^{+} U\right) .
\end{aligned}
$$

The $T$-dependent parameters are obtained by integrating out all non-zero modes and all heavy particles like in (2.1), which is the first part of the Lagrangian (4.3). Thus one has to specify the field content and the SUSY-breaking parameters of the model. The simplest choice is the minimal supersymmetric standard model (MSSM) [26] without universality for the top scalar SUSY-breaking masses. The partner of the left-handed top with a SUSY-breaking mass $m_{Q}^{2}$ should be heavy in order not to contribute too much to $\Delta \rho$. 


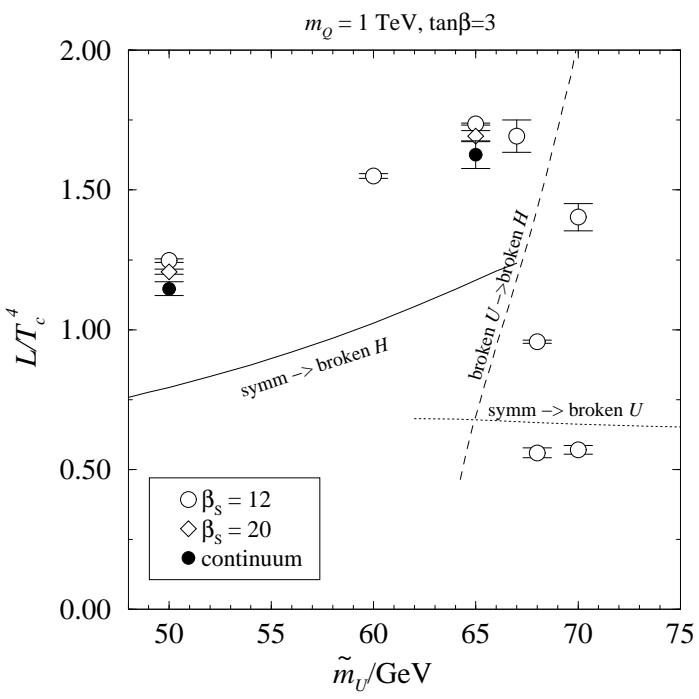

Figure 8: Latent heat at $T_{c}$ in dependence of mass parameter $\tilde{m}_{U}$ calculated on the lattice in ref. [33] compared to the analytic results of ref. [31].

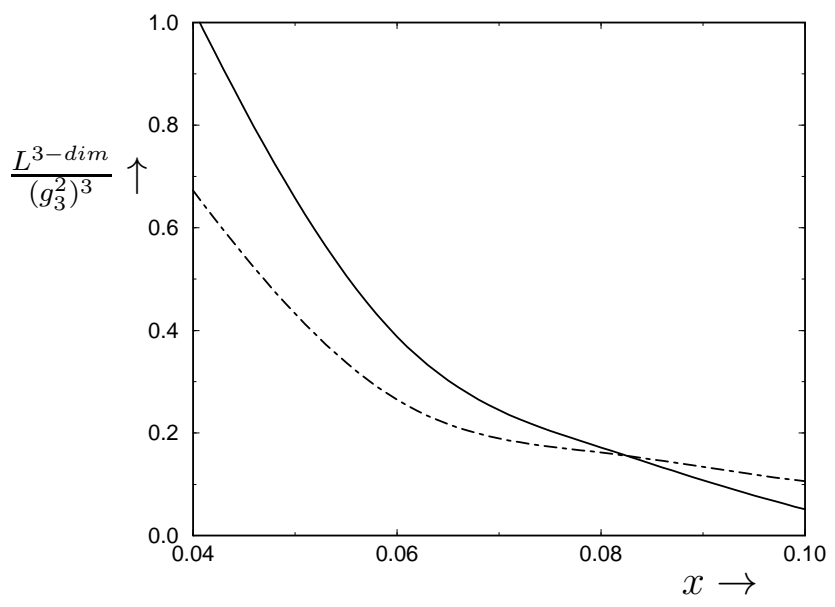

Figure 9: Three dimensional latent heat in dependence of $x$ calculated from the potential (3.2) (full line) compared to the result of ordinary 1-loop perturbation theory (dashed-dotted line). 
Two-loop calculations with (4.3) have shown that one can indeed obtain $v\left(T_{c}\right) / T_{c} \gtrsim 1$ even for lightest Higgs masses as big as $105 \mathrm{GeV}$ [31]. The parameter space is enlarged [32] if one allows for stop $_{R^{-}}$stop $_{L}$ mixing with a parameter $\tilde{A}_{t}=A_{t}+\mu \ldots$. (Both parameters $\mu$ and $A_{t}$ are important in the discussion of CPviolations in the wall.) Ref. [32] uses an improved 4-dimensional one-loop effective potential at high temperatures and still agrees well with the special case considered in 31 .

For large enough negative $m_{U}^{2}=-\tilde{m}_{U}^{2}$ one even obtains [31, 32] a two-stage phase transition with an intermediate stop condensate $\left\langle U^{+} U\right\rangle$. This is only acceptable if the transition rate which is rapidly decreasing with increasing $\tilde{m}_{U}^{2}$ still allows to return from the stop phase to the Higgs phase. In the former phase one has a situation analogous to the Higgs phase, in particular massive $S U(3)$ gauge bosons.

Recent lattice calculations confirm the perturbative results surprisingly well 33] (fig. 8) - though there are also significant deviations. In particular the PT turned out to be more strongly first order - the latent heat and $v\left(T_{c}\right) / T_{c}$ are larger than in the perturbative result. We can understand this effect qualitatively with our model for nonperturbative contributions: The effective $x$-value in the Higgs part of (4.3) is much smaller than in the standard model and for these values (fig. 9) the latent heat and $v\left(T_{c}\right) / T_{c}$ are both increased compared to pure perturbation theory. The important additional graphs coming from Lagrangian (4.3) mostly involve $S U(3)$ gluons and the $s{ }_{0}$ both of which do not have $S U(2)_{W}$ interaction, and hence also no nonperturbative effects on this scalet.

\section{NMSSM with a strongly first-order phase tran- sition}

In the effective electroweak potential near the critical temperature a term of type $-\varphi^{3}$ triggers a first-order PT. Up to now we discussed the generation of such terms in 1-loop order of perturbation theory. There is also the possibility to obtain it already on the tree level. An $S U(2)_{W}$-invariant third-order polynomial term in the potential cannot just contain the Higgs(es). Thus one has to enlarge the field content of the SM and also of the MSSM in the case of a supersymmetric theory. The simplest extension of the MSSM, the "next to minimal model" NMSSM [34, 35], contains a further superfield $S$, which is a gauge singlet, in an additional piece of the superpotential

$$
g^{S}=\lambda S H_{1} H_{2}-\frac{k}{3} S^{3}
$$

The soft SUSY breaking term

$$
V^{S}=A_{\lambda} \lambda S H_{1} H_{2}-\frac{k}{3} A_{k} S^{3}
$$

${ }^{\dagger}$ This remark may be used to consider a hybrid model combined out of lattice and perturbative calculations. 
has the desired " $\varphi$ " form [37] if $S$ is on the same level as the $H_{i}$. The superpotential (5.1) has the virtue to avoid the $\mu$-term $g^{\mu}=\mu H_{1} H_{2}$ with its fine-tuning problem because this term automatically arises after the singlet field aquires a vev. However, because of its $Z_{3}$ symmetry it suffers from the well-known domain wall problem [38. It turns out that the NMSSM with just (5.1) and (5.2) besides having the domain wall problem also is unable to produce a phase transition in $\langle S\rangle$ and $<H>$ simultaneously, which requires $\langle S\rangle$ and $\langle H\rangle$ to be of the same order of magnitude. With a very large $<S>$ f one would first obtain a PT in $\langle S\rangle$ and afterwards the ordinary MSSM PT in some Higgs field combination, which is not what we want. We thus as in ref. [39] choose the superpotentials

$$
g=g^{S}+\mu H_{1} H_{2}-r S
$$

Different from work 44 more than a decade ago we keep the full parameter space of the model only restricted by universal SUSY breaking at the GUT scale. In the latter we differ from ref. [39] where the parameters were fixed at the electroweak scale without such a criterion. Besides the well-known gauge couplings in the $D$ terms we then have the parameters $\lambda, k, \mu, r$ in the superpotential and for the SUSY breaking a universal scalar mass squared $m_{0}^{2}$, a common gaugino mass $M_{0}$, as well as an analytic mass term $B_{0}$ for the Higgses and a universal trilinear scalar coupling $A_{0}$ corresponding to the second and third power terms in the superpotential, respectively.

Besides the tree potential and 1-loop Coleman-Weinberg corrections we include 1-loop plasma masses for the $H_{i}$ and $S$ fields and the 1-loop " $\varphi$ " terms discussed in previous chapters which, however, now in general are small compared to the tree term (5.2). The most important finite temperature contributions come from the top quark and the gauge bosons, but in some parts of the parameter space the stops, charginos and neutralinos may become rather light and therefore are also included in the effective potential $V_{T}\left(H_{1}, H_{2}, S\right)$.

Having at hand the potential we are interested in, a rather natural procedure would be as follows: (Randomly) choose a set of the GUT scale parameters listed above. Then use the (1-loop) renormalization group equations 45 to evolve the parameters down to the weak scale and minimize the $\mathrm{T}=0$ effective potential in order to study the electroweak symmetry breaking. Of course, to reproduce the physical Z-boson mass $M_{Z}$ a rescaling of all the (unknown) dimensionful parameters is necessary. But in the very most number of cases after this rescaling there appear some unobserved light particles in the spectrum, so one has to try the next set of parameters and this whole "shot-gun" procedure is very inefficient.

\footnotetext{
${ }^{\ddagger}$ It was shown in ref. [35] that in the case of universal soft SUSY breaking at the GUT scale the singlet vev has to be larger than $1 \mathrm{TeV}$ to prevent the appearence of not observed light particles in the spectrum. Similar results were obtained for certain non-universal soft terms [36].

${ }^{\S}$ We are aware that removing the cosmologically problematic $Z_{3}$ symmetry after inclusion of non-renormalizable interactions may reintroduce quadratically divergent singlet tadpoles which can destabilize the electroweak scale [40]. But there are some suggestions in the literature how to prevent these potentially dangerous diagrams [41] or even use them in a constructive way as a tool for model building 42,43 .
} 
Instead we fix the $\mathrm{T}=0$ electroweak minimum determined by $M_{Z}, \tan \beta=v_{2} / v_{1}$ and $\langle S\rangle$ in addition to the parameters $\lambda, k, m_{0}^{2}, M_{0}, A_{0}$ while $\mu, r, B_{0}$ remain unspecified. The important thing is that the latter do not enter the 1-loop renormalization group equations for $\mu, r$ and the soft parameters with exception of $B$ so we can calculate all parameters of the effective potential at the weak scale except $\mu, r$ and $B$ which we determine by applying the minimization conditions

$$
\frac{\partial V_{T=0}\left(H_{1}, H_{2}, S\right)}{\partial H_{i}}=0 \quad, \quad \frac{\partial V_{T=0}\left(H_{1}, H_{2}, S\right)}{\partial S}=0 \quad .
$$

Because of the complicated 1-loop corrections these equations cannot be solved analytically, but an iterative numerical solution taking the tree level solution as starting values is possible. Of course, whether the postulated minimum $\left(M_{Z}, \tan \beta,<S>\right)$ is indeed the global minimum has to be checked explicitly and constrains the parameter space of the model. Using this procedure we are left with the seven parameters"I]

$$
\tan \beta,<S>, \lambda, k, m_{0}^{2}, M_{0}, A_{0}
$$

which still contain a lot of freedom. Fortunately, not all parameters are equally important with respect to the strength of the PT: Of most interest are the gaugino mass $M_{0}$ and the trilinear scalar coupling $A_{0}$, as they determine the coefficients $A_{\lambda}$ and $A_{k}$ of the " $\varphi$ " -terms in eq. (5.2). Therefore we will study the plane of these parameters while keeping the others fixed. To maximize the lightest CP-even Higgs mass $M_{h} \tan \beta$ should be taken large while $\lambda$ should be kept small. As stated above, a strong PT can only be expected, if $\left\langle S>\sim M_{Z}\right.$ which requires $k$ to be not too small because of $\left\langle S>\sim \frac{A_{k}}{k}\right.$. The remaining parameter $m_{0}^{2}$ only influences the masses of the additional Higgs bosons which we have chosen heavy.

An example of a scan in the $M_{0}-A_{0}$ plane is shown in fig. (10) where we fixed the remaining parameters according to the remarks before as $<S>=100 \mathrm{GeV}$, $\tan \beta=5, \lambda=0.05, k=0.4$ and $m_{0}=200 \mathrm{GeV}$. There are several constraints on the parameter space: First of all, the minimum postulated in the elimination procedure discussed above has to be the global minimum which leads to the lower bound on $A_{0}$ in fig. (10). To prevent the appearence of a chargino with mass smaller than $80 \mathrm{GeV}$ the gaugino mass $M_{0}$ has to be larger than $100 \mathrm{GeV}$ corresponding to the vertical line in the plot. Finally, we require the lightest Higgs mass $M_{h}$ to be larger than $65 \mathrm{GeV}$ which leads to the upper bound on $A_{0}$ in fig. (10)川 Compared with the current LEP data on SM-like Higgs bosons this may seem to be a rather low value but one has to keep in mind that the lightest "Higgs" state in this model always has some singlet component which even dominates in the region above the dashed line. Therefore the experimental constraints on $M_{h}$ are somewhat relaxed.

In order to investigate the strength of the PT we determine the critical temperature $T_{c}$ where there exist two degenerate minima in $V_{T}\left(H_{1}, H_{2}, S\right)$, a broken

${ }^{\top}$ Additionally, we require the top quark mass $M_{t o p}=175 \mathrm{GeV}$ which allows us to fix the top Yukawa coupling as a function of $\tan \beta$. All the other Yukawa couplings are neglected which is only justified in the regime $\tan \beta \lesssim 10$.

"Note that this also implies an upper bound on the gaugino mass depending on the remaining parameters. 


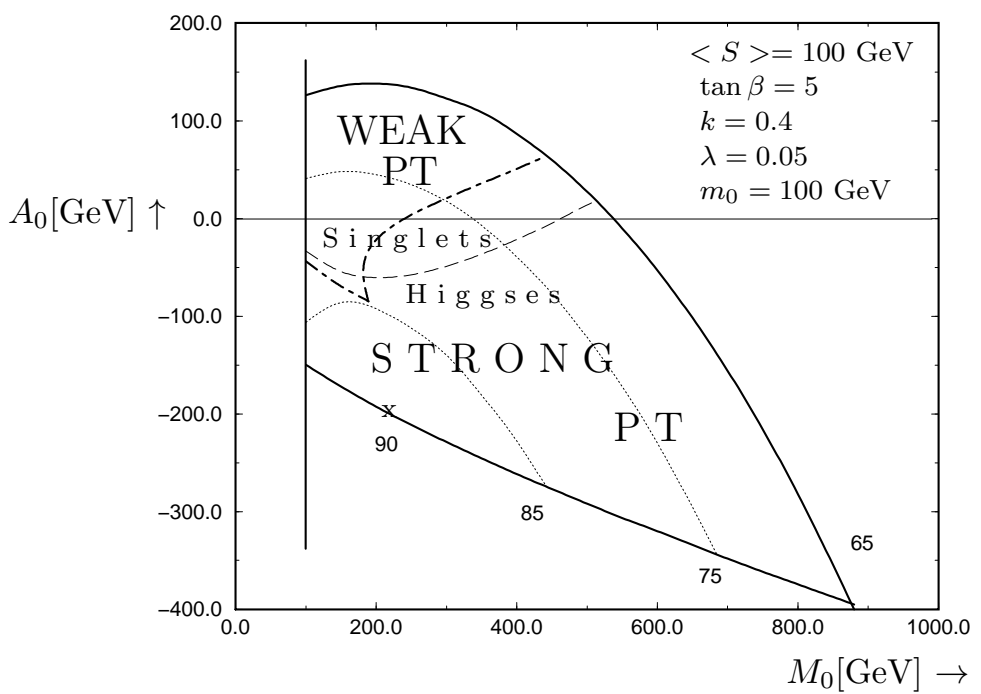

Figure 10: Scan of the $M_{0}-A_{0}$ plane where the remaining parameters are fixed. The full line surrounds the phenomenologically viable part of the parameter space. The dotted lines are curves of constant lightest Higgs mass $(75$ and $85 \mathrm{GeV}$ ). The dashed line indicates the region where the lightest Higgs is predominantely a singlet. The dashed-dotted line separates the regions of strong $\left(v_{c} / T_{c} \gtrsim 1\right)$ and weak PT.

minimum with $<H_{i}>\neq 0$ and a symmetric one with $\left\langle H_{i}>=0\right.$. For the previously discussed set of parameters the results are again summarized in fig. (10). There the dashed-dotted line separates the region with a weak PT from the region where the baryon number washout criterium $v_{c} / T_{c} \gtrsim 1$ is fulfilled. One clearly sees that most of the parameter space is indeed compatible with electroweak baryogenesis. Interestingly enough, the region where the Higgs mass is maximized $\left(M_{h} \sim 90\right.$ $\mathrm{GeV}$ ) is not excluded. Let us again stress that the situation drastically changes if we increase the singlet vev to e. g. $\langle S\rangle=300 \mathrm{GeV}$ while decreasing $k$ in order to obtain similar values of $M_{h}$. Then only a small range of values of $A_{0}$ just above its lower bound allows a strong PT and most of the parameter space leads to erasure of the baryon asymmetry.

In the previous example the maximal value of the Higgs mass is $90 \mathrm{GeV}$ but one can reach much higher values. By choosing $\tan \beta=10 M_{h}=100 \mathrm{GeV}$ can be obtained and still $v_{c} / T_{c} \gtrsim 1$ can be fulfilled. Increasing the singlet vev to e. g. $\langle S\rangle=250$ $\mathrm{GeV}$ allows the even larger value of $M_{h}=115 \mathrm{GeV}$ without violating the washout criterium. But with larger $\langle S\rangle$ the amount of fine-tuning of $A_{0}$ increases and there is the danger of metastability since the PT requires thermal tunneling over a rather high tree barrier.

\footnotetext{
${ }^{* *}$ The singlet vev is different from zero even in the symmetric minimum.
} 


\section{Concluding remarks; baryogenesis in the SUSY electroweak phase transition}

Having found a model and a set of parameters where the electroweak PT is strong enough to avoid sphaleron erasure in the Higgs phase of a previously generated baryon asymmetry, we are just at the beginning and not at the end of the story: One now has to develop a consistent picture of baryogenesis [46] [53].

First one has to derive the shape of the bubble wall of the critical bubble [54, 55]. The corresponding action determines the transition probability and - together with the Hubble parameter - the degree of supercooling and the nucleation temperature. Similarly the friction generated by scattering processes at the bubble wall determines the shape of a stationary expanding bubble 48 .

Furthermore, CP-violation in the bubble wall formed by a spatially varying Higgs-field combination has to be discussed 449- 52]. Different from the SM in SUSY models the phases of the Higgs-field couplings $\mu$ and $A_{t}$ cannot be defined away. CP could be broken explicitly or spontaneously by Higgs condensates with a phase. The latter might only happen in some temperature interval. This is very attractive since it relaxes dangerous upper bounds by an experimentally allowed neutron electric dipole moment [52] (one also needs some explicit CP-breaking in order to remove a sign ambiguity in the spontaneous breaking).

In variants of the favourite "charge transport mechanism" [53] the scattering (transmission/reflection) of the particles in the plasma (most important are Higgsinos, gauginos, stop) on the bubble wall generates a current of chiral charge diffusing in the symmetric phase in front of the expanding bubble and is transmitted into a $B+L$ asymmetry by the "hot" (unsuppressed) sphaleron processes during the time before the Higgs phase bubble takes over. The bubble wall in new calculations 566[60) is favoured to be thick (i.e. bigger than the mean free path of the scattering particles) and is moving slowly ( $\sim c / 10$, "deflagration"). Recently it was stressed in the literature [56, 57, 60] that one should not separate scattering at the bubble wall and diffusion in the symmetric phase, but deal with both simultaneously in the framework of quantum Boltzmann equations. The outcome is still controversely discussed.

Concluding we can say that the strength of the electroweak phase transition in specific models like the one discussed here can be determined reliably using a mix of perturbative theory and lattice work, supplemented by a qualitative analytic picture. If the PT is predicted by perturbative calculations to be strongly first-order, this is not changed by nonperturbative effects. In the MSSM with a "light" $s t o p_{R}$ and in a broad parameter range of the NMSSM with $\mu \neq 0$ a strongly first-order PT with $v\left(T_{c}\right) / T_{c} \gtrsim 1$ is possible even at Higgs masses as big as $100 \mathrm{GeV}$ (and even higher?).

\footnotetext{
${ }^{\dagger \dagger}$ In case of the NMSSM there may exist some parts of the parameter space where the wall turns out to be thin.
} 


\section{Acknowledgement}

We would like to thank P. John, O. Philipsen and M. Reuter for useful discussions. This work was supported in part by the TMR network Finite Temperature Phase Transitions in Particle Physics, EU contract no. ERBFMRXCT97-0122.

\section{References}

[1] V. A. Kuzmin, V. A. Rubakov, and M. E. Shaposhnikov, Phys. Lett. B155 (1985) 36; M. E. Shaposhnikov, Nucl. Phys. B287 (1987) 757.

[2] V. A. Rubakov and M. E. Shaposhnikov, Usp. Fiz. Nauk 166 (1996) 493 hepph/9603208; M. E. Shaposhnikov, CERN-TH/96-280 hep-ph/9610247 and further references quoted therein.

[3] E. Kolb, Turner, The Early Universe and references therein.

[4] D. A. Kirzhnits, A. D. Linde, Phys. Lett. B42 (1972) 471.

[5] see e.g. G. W. Anderson, L. J. Hall, Phys. Rev. D45 (1992) 2685.

[6] K. Kajantie, M. Laine, K. Rummukainen, and M. Shaposhnikov, Nucl. Phys. B466 (1996) 189; Phys. Rev. Lett. 77 (1996) 2887.

[7] K. Farakos, K. Kajantie, K. Rummukainen, and M. Shaposhnikov, Nucl. Phys. B425 (1994) 67; K. Kajantie, M. Laine, K. Rummukainen, and M. Shaposhnikov, Nucl. Phys. B458 (1996) 90.

[8] E. Braaten, A. Nieto, Phys. Rev. D51 (1995) 6990; 53 (1996) 3421.

[9] P. Ginsparg, Nucl. Phys. B170 (1980) 388;

T. Appelquist and R. Pisarski, Phys. Rev. D23 (1981) 2305;

S. Nadkarni, Phys. Rev. D27 (1983) 917.

[10] K. Kajantie, M. Laine, K. Rummukainen, M. Shaposhnikov, Nucl. Phys. B466 (1996) 189; Phys. Rev. Lett. 77 (1996) 2887.

[11] E.-M. Ilgenfritz, J. Kripfganz, H. Perlt, A. Schiller, Phys. Lett. B356 (1995) 561; M. Gürtler, E. M. Ilgenfritz, J. Kripfganz, H. Perlt, A. Schiller, Nucl. Phys. Proc. Supp. 49 (1996) 312; Nucl. Phys. B483 (1997) 383; M. Gürtler, E. M. Ilgenfritz, A. Schiller, Phys. Rev. D56 (1997) 3888; Eur. Phys. J. C1 (1998) 363.

[12] B. Bergerhoff and C. Wetterich, Nucl. Phys. B440 (1995) 171, hep-ph/9611462;

N. Tetradis, Nucl. Phys. B488 (1997) 92;

J. Berges, N. Tetradis, C. Wetterich, Phys. Lett. B393 (1997) 387.

[13] S. J. Huber, A. Laser, M. Reuter, M. G. Schmidt, HD-THEP-98-16, MZ-TH/9815, hep-ph/9804441. 
[14] K. Rummukainen, M. Tsypin, K. Kajantie, M. Laine, M. Shaposhnikov, CERNTH/98-08, NORDITA-98/30HE, hep-lat/9805013.

[15] J. Kripfganz, A. Laser, M. G. Schmidt, Z. Physik C73 (1997) 353.

[16] Z. Fodor, J. Hein, K. Jansen, A. Jaster, I. Montray, F. Czikor, Phys. Lett. 334 (1994) 405.

[17] F. Czikor, Z. Fodor, J. Hein, J. Heitger, Phys. Lett. 357 (1995) 156.

[18] O. Philipsen, M. Teper, H. Wittig, Nucl. Phys. B469 (1996) 445; Nucl. Phys. B528 (1998) 379.

[19] H. G. Dosch, J. Kripfganz, A. Laser, M. G. Schmidt, Phys. Lett. B365 (1996) 213; Nucl. Phys. B507 (1997) 519.

[20] Y. A. Simonov, Phys. Lett. B266 (1989) 151.

[21] H. G. Dosch, Phys. Lett. B190 (1987) 177;

H. G. Dosch, Y. A. Simonov, Phys. Lett. B205 (1988) 339.

[22] H. G. Dosch, Prog. Part. Nucl. Phys. 33 (1994) 121;

Y. A. Simonov, Schladming Lectures 1996 and further references therein;

O. Nachtmann, Schladming Lectures 1996.

[23] M. Laine, O. Philipsen, Nucl. Phys. B523 (1998) 267;

M. Ilgenfritz, private communication.

[24] M. Carena, M. Quirós, and C. E. M. Wagner, Phys. Lett. B380 (1996) 81.

[25] D. Dellepine, J.-M. Gérard, R. Gonzales Felipe, and J. Weyers, Phys. Lett. B386 (1996) 183.

[26] S. Myint, Phys. Lett. B287 (1992) 325; G. F. Giudice, Phys. Rev. D45 (1992); J. R. Espinosa, M. Quirós, F. Zwirner, Phys. Lett. B307 (1993) 106; A. Brignole, J. R. Espinosa, M. Quirós, F. Zwirner, Phys. Lett. B324 (1994) 181.

[27] M. Losada, Phys. Rev. D56 (1997) 2893; G. R. Farrar and M. Losada, Phys. Lett. B406 (1997) 234.

[28] M. Laine, Nucl. Phys. B481 (1996) 43.

[29] J. M. Cline and K. Kainulainen, Nucl. Phys. B482 (1996) 73; 510 (1998) 88.

[30] J. R. Espinosa, Nucl. Phys. B475 (1996) 273.

[31] D. Bödeker, P. John, M. Laine, M. G. Schmidt, Nucl. Phys. B 497 (1997) 387, hep-ph/9612364.

[32] M. Carena, M. Quiros, C. E. M. Wagner, CERN-TH/97-190 hhep-ph/9710401]. 
[33] M. Laine, K. Rummukainen, CERN/TH/98-122, NORDITA-98/29P heplat/9804019; CERN-TH/98-121 hep-ph/9804255.

[34] J. Gunion, H. E. Haber, G. L. Gordon, S. Dawson, The Higgs Hunter's Guide, Addison-Wesley, Reading MA, 1990;

J. Ellis, J. Gunion, H. Haber, L. Roszkoswki, F. Zwirner, Phys. Rev. D39 (1989) 844;

T. Elliot, S. F. King, P. L. White, Phys. Rev. D49 (1994) 2435; Phys. Lett. B305 (1993) 71; B314 (1993) 56.

[35] U. Ellwanger, M. Rausch de Traubenberg, Z. Phys. C53 (1992) 521;

U. Ellwanger, Phys. Lett. B303 (1993) 271;

U. Ellwanger, M. Rausch de Traubenberg, C. Savoy, Z. Phys. C67 (1995) 665.

[36] P. Brax, U. Ellwanger, C. A. Savoy, Phys. Lett. B347 (1995) 229.

[37] M. Pietroni, Nucl. Phys. B402 (1993) 27.

[38] S. A. Abel, S. Sarkar, P. L. White, Nucl. Phys. B454 (1995) 663.

[39] A. T. Davies, C. D. Froggat, R. G. Moorhouse Phys. Lett. B372 (1996) 88.

[40] U. Ellwanger, Phys. Lett. B133 (1983) 187;

J. Bagger, E. Poppitz, L. Randall Nucl. Phys. B455 (1995) 59.

[41] S. A. Abel, ULB-TH-93/03, hep-ph/9603301.

[42] U. Ellwanger, Phys. Lett. B349 (1995) 57;

H. P. Nilles, N. Polonsky, Phys. Lett. B412 (1997) 69.

[43] Kolda, Pokorski, Polonsky Phys. Rev. Lett. 80 (1998) 5263.

[44] N. Dragon, U. Ellwanger, M. G. Schmidt, Prog. Part. Nucl. Phys. 18 (1987) 1, and ref. quoted there.

[45] J. P. Derendinger, C. A. Savoy, Nucl. Phys. B 237 (1984) 307.

[46] B. H. Liu, L. McLerran, N. Turok, Phys. Rev. D46 (1992) 2668;

P. Huet, K. Kajantie, R. G. Leigh, B. H. Liu, L. McLerran Phys. Rev. D48 (1993) 2477.

[47] M. Joyce, T. Prokopec, N. Turok, Phys. Rev. D53 (1993) 2930, 2958.

[48] G. D. Moore, T. Prokopec, Phys. Rev. D52 (1995) 7182, Phys. Rev. Lett. 75 (1995) 777.

[49] P. Huet, A. E. Nelson, Phys. Rev. D53 (1996) 4578.

[50] J. M. Cline, K. Kanulainen, A. P. Visher, Phys. Rev. D54 (1996) 2451. 
[51] K. Funakubo, A. Kakuto, S. Otsuki, F. Toyoda, Prog. Theor. Phys. 95 (1996) 929;

K. Funakubo, SAGA-HE-108, hep-ph/9608358 and ref. therein;

K. Funakubo, A. Kakuto, S. Otsuki, F. Toyoda, Prog. Theor. Phys. 98 (1997) 427, hep-ph/9802276.

[52] M. Aoki, N. Oskimo, A. Sugamoto, Prog. Theor. Phys. 98 (1997) 1179, hep$\mathrm{ph} / 9706287$.

[53] A. G. Cohen, D. B. Kaplan, A. E. Nelson, Phys. Lett. B245 (1990) 561, Nucl. Phys. B349 (1991) 727;

A. E. Nelson, D. B. Kaplan, A. G. Cohen, Nucl. Phys. B373 (1992) 453;

D. B. Kaplan, hep-ph/9503360 and ref. therein;

M. Joyce, T. Prokopec, N. Turok, Phys. Lett. B338 (1994) 269, Phys. Rev. Lett. 75 (1995) 1695.

[54] K. Enquist, J. Ignatius, K. Kajantie, K. Rummukainen, Phys. Rev. D45 (1992) 3415.

[55] J. Ignatius, K. Kajantie, H. Kurki-Suoni, M. Laine, Phys. Rev. D49 (1994) 3854 .

[56] M. Carena, M. Quiros, A. Riotto, I. Vilja, C. E. M. Wagner, Nucl. Phys. B503 (1997) 387.

[57] A. Riotto, OUTP-97-43-P, hep-ph/9709286; Nucl. Phys. B518 (1998) 339; OUTP-98-23-P, hep-ph/9803357.

[58] J. M. Cline, M. Joyce, K. Kanulainen, Phys. Lett. B417 (1998) 79.

[59] H. Davoudiasl, K. Rajagopal, E. Westphal, Nucl. Phys. B515 (1998) 384.

[60] M. Joyce, K. Kanulainen, T. Prokopec, CAPP-98, CERN June 1998. 